\title{
Non Convulsive Status Epilepticus: An Elusive Diagnosis
}

\author{
Rebeca 0. Millan-Guerrero1, Sara Isais-Millan2 ${ }^{2 *}$ \\ ${ }^{1}$ Department of Neurology, Unidad de Investigación HGZ 1 IMSS, Mexico City, Mexico \\ ${ }^{2}$ Movement Disorders Clinic, Instituto Nacional de Neurologia y Neurocirugia MVS, Mexico City, Mexico \\ Email: *millanrebeca@hotmail.com
}

Received 22 December 2015; accepted 13 February 2016; published 16 February 2016

Copyright (C) 2016 by authors and Scientific Research Publishing Inc.

This work is licensed under the Creative Commons Attribution International License (CC BY).

http://creativecommons.org/licenses/by/4.0/

(c) (i) Open Access

\section{Abstract}

Absence status is the most common form of non-convulsive status epilepticus and is characterized by confusion with varying degrees of memory loss and cognitive impairment. Patients and Method: Three children were sent to neurological consultation due to behavioral alterations and a prolonged confused state; they were hospitalized and treated with sodium diphenylhydantoinate (DPH) IV at a dose of $10 \mathrm{mg} / \mathrm{Kg}$. Results: The duration of symptoms varied from 6 months to 10 days. All three patients presented with global mental alterations, showing slowness in response and action. The electroencephalogram showed a pattern of slow, generalized stem and poly-stemwavelengths of $3-4 \mathrm{~Hz}$, which were registered for one hour. After the DPH bolus, the attack spontaneously ended in the 3 patients and upon examination all three presented with amnesia of the events occurring during the attack. In the follow-up, two of the patients did not experience further episodes and they showed normal scholastic achievement. The third patient however, after suffering a 6-month status epilepticus, failed the school year and finished his elementary education until the age of 15, experiencing similar difficulties with his secondary education. Discussion: Nonconvulsive status epilepticus is more difficult to diagnose mainly because the manifestations are predominantly psychiatric and can be confused with other diseases or with an overdose of anticonvulsive drugs. A prolonged state of mental confusion, with no other explanation, should alert the attending physician to take an electroencephalogram in order to confirm the diagnosis. In our patients, DPH immediately controlled paroxysmal activity. We can therefore conclude that the problem is not in the treatment, but rather in making the correct diagnosis.

\section{Keywords}

Non-Convulsive Status Epilepticus, Absence Status Epilepticus, Morbidity, Cognitive Effects

\footnotetext{
${ }^{*}$ Corresponding author.
} 


\section{Introduction}

Non-convulsive generalized status epilepticus (GNCSE) is defined as a state lasting more than 1 hour, characterized by acute onset of the confusional state with memory function defect to a varying degree [1] [2]. However, Non-Convulsive Status Epilepticus (NCSE) was not clearly established as an entity until the introduction of the EEG in the 1940s. In 1962 an international symposium subdivided SE into convulsive and non-convulsive [3], the latter constituting about $14 \%$ - 25\% of all SE cases [3]-[7].

Generalized non-convulsive status is known by various names: Spike-wave stupor, epileptic twilight state, petit mal status, and absence status. Absence NCSE will be defined as episodes in which a patient exhibits a prolonged "twilight" state or a cyclic waxing and waning of responsiveness [8], fluctuating confusion of varying intensity, slowness in behavior and mentation and sometimes stupor (or coma), associated with bilateral myoclonia in one half of the patients [9]-[12]. The EEG exhibits generalized continuous or nearly continuous epileptiform activity in the form of bilaterally synchronous spike-wave discharges (SWDs) or more complex discharges during periods of reduced responsiveness. The neuronal network generating these seizures includes the thalamic and neocortical populations [13]. NCSE is difficult to diagnose because it presents in protean ways and lacks overt convulsive activity [14]. The definitive diagnosis is made with an EEG.

The objective of this study was to describe the clinical and electroencephalographic characteristics of long generalized non-convulsive status epilepticus.

\section{Patients and Method}

Three children with a history of generalized tonic-clonic seizures controlled with diphenylhydantoinate between 1986 and 1988 were studied. In all of them the etiology of epilepsy was unknown, none had the Lennox Gastaud syndrome and they had normal intellectual development. They were attending primary school with adequate academic performance. Without precipitating factors they were sent to external consultation due to behavioral alterations and a prolonged confused state, after having been seen by different specialists and having undergone unsuccessful treatment modifications. Criteria for inclusion in the study were 1) one or more spells of GNCSE observed by us, and 2) rapid return to near-normal behavior and mentation simultaneous with termination of EEG epileptiformactivity.

Case 1: A seven-year-old boy, presenting with disorientation and attention-deficit problems at school. Description of status: patient showed confusion and slowness of responses, a catatonic-like state with markedly reduced motor activity; slow and ataxic gait often requiring assistance, he could carry out simple activities, such as eating or washing. Spontaneous speech was non-fluent, with a long latency of response and perseveration. He could intermittently respond to request or remember a brief phrase for 3 minutes, do simple calculations, name objects, repeat a phrase, or attempt to copy a simple drawing. These acts (which often required repeated commands from the examiner) were generally carried out slowly and imperfectly. Neurologic examination findings during the attack were normal. The average duration of status was over a period of six months.

Case 2: A ten-year-old girl presenting with confusion, non-fluent spontaneous speech, repetitive movements with automatisms and slowness of responses, slowness in walking speed and changes in conduct over a period of fifteen days.

Case 3: A twelve-year-old boy presenting with behavioral slowness and mental confusion over a period of 10 days. Recordings were made on an 8-channel Grass electroencephalograph. Electrodes were placed according to the international 10 - 20 system. Paper speed was $30 \mathrm{~mm} / \mathrm{sec}$ and corresponded to a recording time of approximately one hour.

Two parameters were computed in order to quantify the abundance of ictal activity on individual recordings: the mean interval between seizures and a seizure index, defined as the percent time of the total recording occupied by ictal activity. All patients were given complete evaluations, including CT, to determine the possible causes of the epilepsy.

The three patients were hospitalized and treated with sodium diphenylhydantoinate (DPH) IV at a dose of 10 $\mathrm{mg} / \mathrm{Kg}$. EEGs were repeated at 24 hours and the patients continued to receive a DPH maintenance dosage of 4 $\mathrm{mg} / \mathrm{Kg}$ PO. There was a ten-year patient follow-up, including yearly EEGs.

\section{Results}

The average duration of status varied from 6 months to 10 days. Mental alteration was observed in each patient, 
varying in degree from medium disorientation to confusion. All three patients presented with global mental alterations, showing slowness in response and actions. In all three cases, the EEG taken during the attack showed predominantly symmetrical generalized ictal activity in the form of continuous spike-wave or polyspike-wave discharges with little variation in waveform or frequency (Figure 1 and Figure 2). This activity was registered for one hour. The frequency of spike-wave complexes ranged from approximately 1 to $3 \mathrm{~Hz}$. After a DPH bolus, the attack spontaneously ended in the three patients and upon examination all three presented with amnesia of the events occurring during the attack. Behavior alertness and EEG returned to near normal within 10 minutes of IV injection of DPH (Figure 3). The three patients took valproic acid (in addition to phenytoin or carbamazepine) in dosages maintaining therapeutic blood levels. During the 5-year follow-up, the Case 2 and Case 3 patients did not present with further seizures or similar events and they showed normal scholastic achievement. There was no evidence of long-term deterioration of intellect, memory or behavior.

The Case 1 patient, presenting with status epilepticus for 6 months, received follow-up until he was 20 years old. He failed the school year and finished his elementary education until the age of 15, experiencing similar difficulties with his secondary education. Follow-up EEGs demonstrated occasional slow waves at the frontal area (Figure 4).

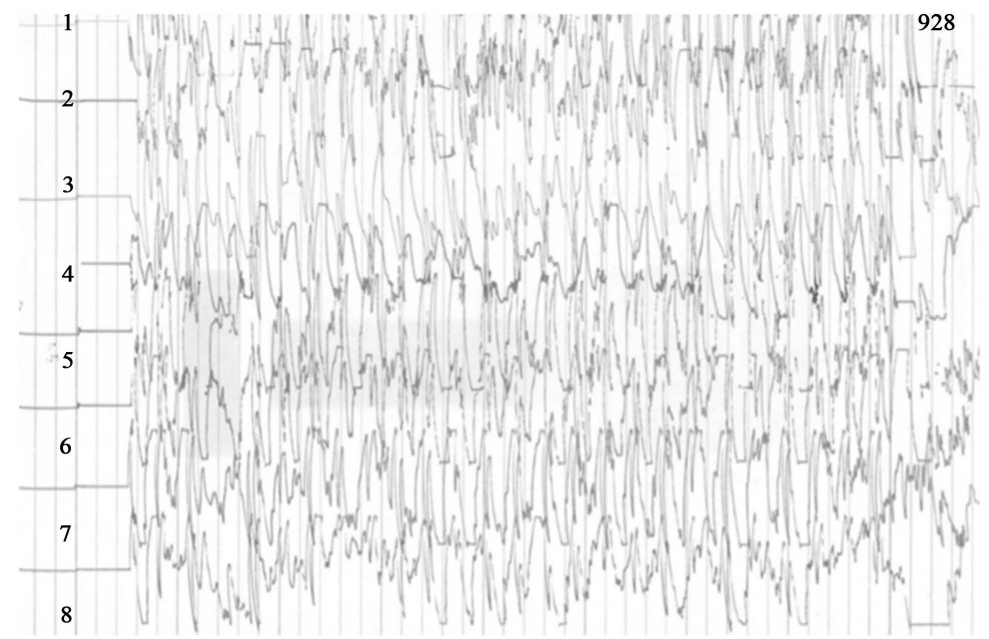

Figure 1. Patient 1: EEG-recording during ictal phase, rhythmic and stereotyped spike, polispike and spike-wave generalized discharges. This vertical calibration bar is $50 \mu \mathrm{V}$. Time interval between vertical dividing lines is 1 second.

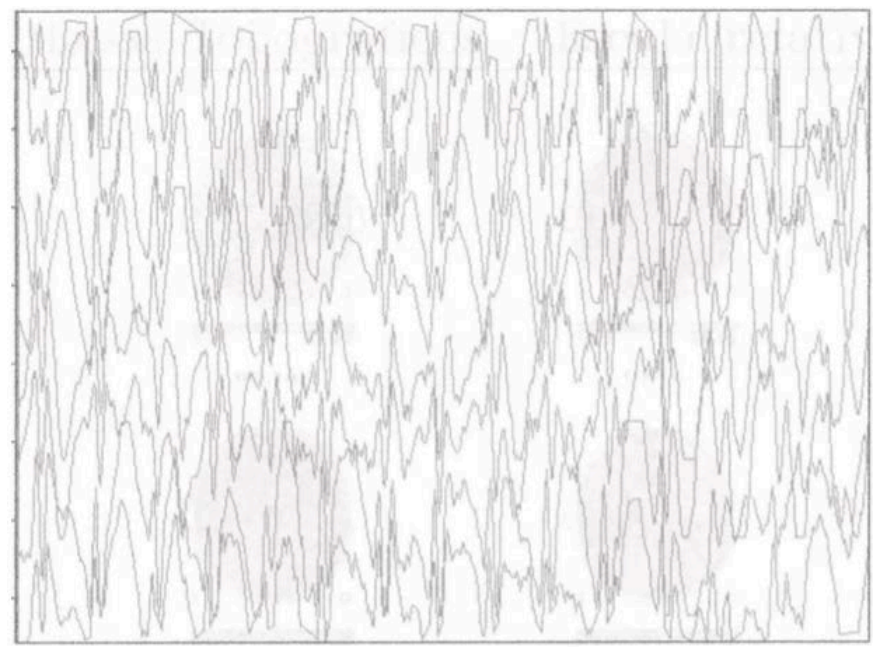

Figure 2. Patient 2: This recording shows generalized nonconvulsive status epilepticus with spikewave discharges occur from $3 / \mathrm{s}$. 


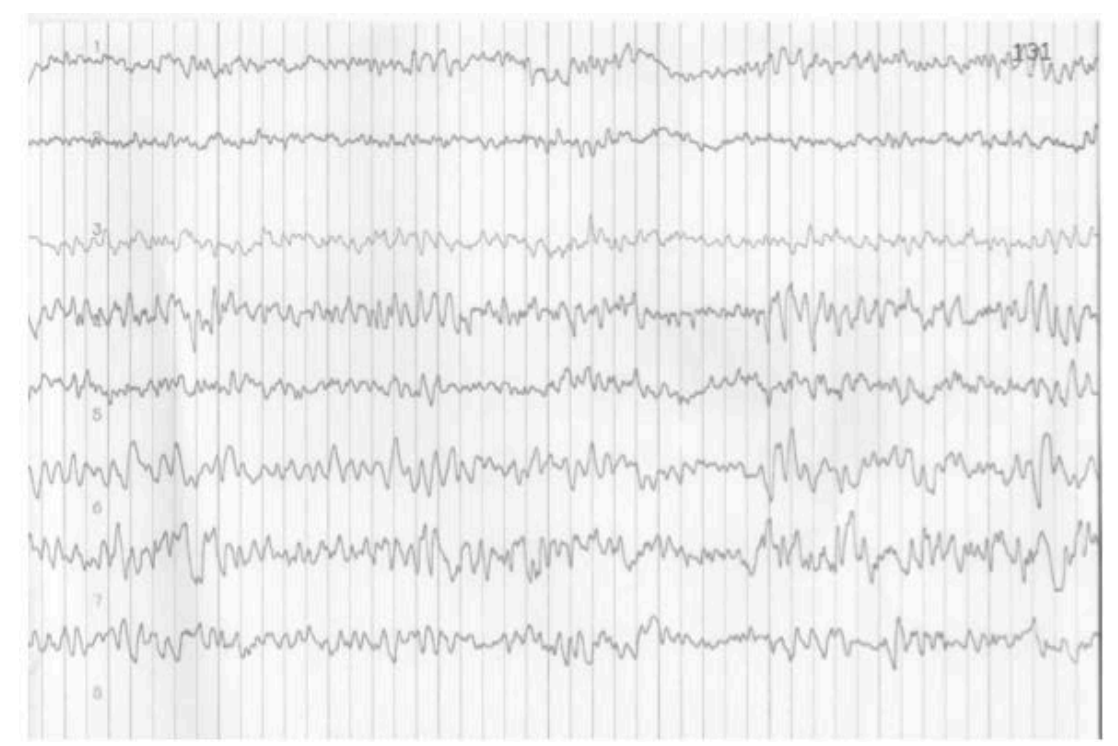

Figure 3. Patient 1: Routine EEG performed 3 days postictally after receiving $300 \mathrm{mg}$ de DPH, shown diffuse irregular theta-delta activity, spike-wave, and sharp-wave discharges.

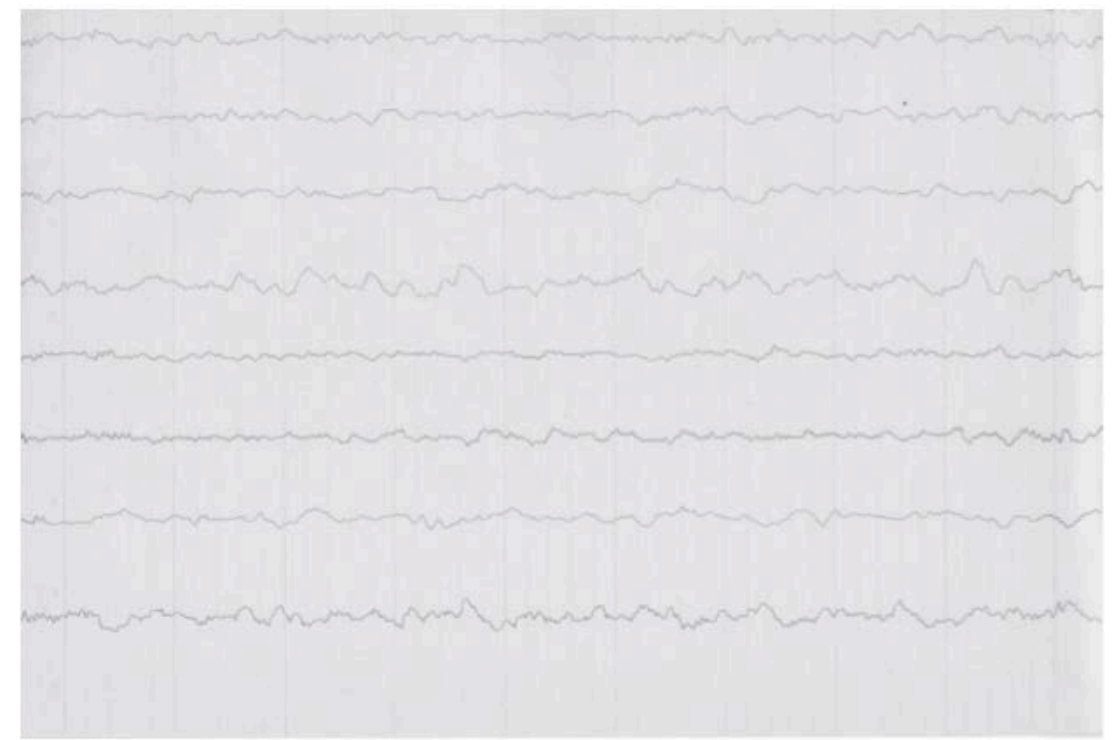

Figure 4. Patient 1: Evolution of generalized nonconvulsive status epilepticus in a 9 yearold boy generalized, low to medium-voltage theta-delta activity.

\section{Discussion}

Non-convulsive status epilepticus (NCSE) has been considered a rare form of SE [15], which is a diagnostic problem unassociated with serious morbidity and death [1]. Non-convulsive status epilepticus is more difficult to diagnose than the convulsive status, mainly because the manifestations are predominantly psychiatric and can be confused with other diseases or with an overdose of anti-convulsive drugs, especially when dealing with patients having a history of epilepsy with a different pattern [16] [17]. The criteria used to define GNCSE were [1] [5]: 1) Prolonged change of consciousness or behavioral function (greater than 30 minutes). 2) Generalized epileptic EEG abnormality that is definitively changed from the preictal state. 3) A prompt and observable pharmacological effect of an intravenous antiepileptic drug on both ictal EEG and clinical manifestations of the status.

A prolonged state of mental confusion that persists for a sufficient length of time and that has no other expla- 
nation should alert the attending physician to take an electroencephalogram in order to confirm the diagnosis. The importance of EEG monitoring in the diagnosis and management of GNCSE has been increasingly acknowledged [18] [19]. Because ictal activity may not be associated with clinical manifestations, EEG monitoring is occasionally necessary to diagnose GNCSE. Similarly, when status epilepticus seems under control clinically, electroencephalography is required to ensure that seizure activity has ceased [11].

Treatment and management of patients with non-convulsive status epilepticus has been frequently discussed [20]. IV diazepam has traditionally been the drug of choice for the termination of absence status. There are reports of cases of absence status that were successfully treated by IVphenytoin. In our patients, DPH immediately controlled paroxysmal activity [21].

\section{Conclusion}

We can therefore conclude that the problem is not in treating the disease, but rather in making the correct diagnosis. Although the non-convulsive status has been described as self-limited and benign, this is not really true, because if it persists for a sufficient length of time it can cause serious brain damage, manifesting itself as serious learning disabilities. This might indicate that NCSE in acutely ill patients should be regarded as seriously as Convulsive Status Epilepticus. Therefore, non-convulsive status epilepticus is a diagnosis that must be considered in patients with altered mental status and it must be treated appropriately with anticonvulsants when found. Because the literature is ambiguous and the criteria proposed for non-convulsive status are evidently based on observations of only a few cases, it seems important that new cases are reported to obtain a broader basis for recognition of its clinical and electroencephalographic characteristics. It is also our obligation to make these cases known, so that no patient mistakenly is left in an epileptic state for as long as 6 months, as was the case with our patient who suffered intellectual deterioration, negatively affecting his scholastic development.

\section{References}

[1] Krumholz, A. (1999) Epidemiology and Evidence for Morbidity of Nonconvulsive Status Epilepticus. Journal of Clinical Neurophysiology, 16, 314-322. http://dx.doi.org/10.1097/00004691-199907000-00003

[2] Pollak, L., Gandelman-Marton, R., Margolin, N., Boxer, M. and Blatt, I. (2014) Clinical and Electroencephalographic Findings in Acutely Ill Adults with Non-Convulsive vs Convulsive Status Epilepticus. Acta Neurologica Scandinavica, 129, 405-411. http://dx.doi.org/10.1111/ane.12200

[3] Guberman, A., Cantu-Reyna, G., Stuss, D. and Broughton, R. (1986) Nonconvulsive Generalized Status Epilepticus: Clinical Features, Neuropsychological Testing, and Long-Term Follow-Up. Neurology, 36, 1284-1289. http://dx.doi.org/10.1212/WNL.36.10.1284

[4] Celesia, G.C. (1976) Modern Concepts of Status Epilepticus. JAMA, 235, 1571-1574. http://dx.doi.org/10.1001/jama.1976.03260410027018

[5] Gastaud, H. (1983) Classification of Status Epilepticus. In Delgado-Escueta, A.V., Wasterlain, C.G., Treiman, D.M., Poster, R.J., et al., Eds., Status Epilepticus, Raven Press, New York, 15-35.

[6] Tomson, T., Svanborg, E. and Wedlund, J.E. (1986) Nonconvulsive Status Epilepticus: High Incidence of Complex Partial Status. Epilepsia, 27, 276-285. http://dx.doi.org/10.1111/j.1528-1157.1986.tb03540.x

[7] De Lorenzo, R.J., Watherhouse, E.J., Towne, A.R., et al. (1998) Persistent Nonconvulsive Status Epilepticus after Control of Convulsive Status Epilepticus. Epilepsia, 39, 833-840.

http://dx.doi.org/10.1111/j.1528-1157.1998.tb01177.x

[8] Porter, R.J. and Penry, J.K. (1983) Petit Mal Status. Advances in Neurology, 34, 61-67.

[9] Andermann, F. and Robb, J.P. (1972) Absence Status. A Reappraisal Following Review of Thirty-Eight Patients. Epilepsia, 13, 177-187. http://dx.doi.org/10.1111/j.1528-1157.1972.tb04565.x

[10] Fernández-Torre, J.L., Rebollo, M., Gutiérrez, A., López-Espadas, F. and Hernández-Hernández, M.A. (2012) Nonconvulsive Status Epilepticus in Adults: Electroclinical Differences between Proper and Comatose Forms. Clinical Neurophysiology, 123, 244-251. http://dx.doi.org/10.1016/j.clinph.2011.06.020

[11] Hosford, D. (1999) Animal Models of Nonconvulsive Status Epilepticus. Journal of Clinical Neurophysiology, 16, 306-313. http://dx.doi.org/10.1097/00004691-199907000-00002

[12] Husain, A.M., Horn, G.J. and Jacobson, M.P. (2003) Non-Convulsive Status Epilepticus: Usefulness of Clinical Features in Selecting Patients for Urgent EEG. Journal of Neurology, Neurosurgery \& Psychiatry, 74, 189-191.

http://dx.doi.org/10.1136/jnnp.74.2.189 
[13] Gloor, P., Quesney, L.F. and Zumstein, H. (1977) Pathophysiology of Generalized Penicillin Epilepsy in the Cat: The Role of Cortical and Subcortical Structures. Electroencephalography and Clinical Neurophysiology, 43, 79-94. http://dx.doi.org/10.1016/0013-4694(77)90198-5

[14] Gómez-Ibáñez, A., Urrestarazu, E. and Viteri, C. (2012) Estado epiléptico no convulsivo en el siglo XX1: Clínica, diagnóstico, tratamiento y pronóstico. Revue Neurologique, 54, 105-113.

[15] Gastaut, H. (Ed.) (1975) Handbook of Electroencephalography and Clinical Neurophysiology. Vol. 13A, Eelsevier, Amsterdam, 39-45.

[16] Jordan, K.G. and Hirsch, L.J. (2006) In Nonconvulsive Status Epilepticus (NCSE), Treattoburst-Suppression: Pro and Con. Epilepsia, 47, 41-45. http://dx.doi.org/10.1111/j.1528-1167.2006.00659.x

[17] Hixson, J.D. (2011) When Physicians Disagree: A Case of Nonconvulsive Status Epilepticus. CONTINUUM: Lifelong Learning in Neurology, 17, 1119-1123. http://dx.doi.org/10.1212/01.con.0000407064.27070.d6

[18] Thomas, P., Zifkin, B., Migneco, O., Lebrun, C., Darcourt, J. and Andermann, F. (1999) Nonconvulsive Status Epilepticus of Frontal Origin. Neurology, 52, 1174-1183. http://dx.doi.org/10.1212/WNL.52.6.1174

[19] Maison, F., Reiher, J. and Leduc, C.P. (1991) Retrospective Inventory of EEG Abnormalities in Partial Status Epilepticus. Electroencephalography and Clinical Neurophysiology, 79, 264-270. http://dx.doi.org/10.1016/0013-4694(91)90121-J

[20] Amit, R., Rottem, M. and Yatziv, S. (1986) Termination of Absence Status and Suppression of Inter-Ictal Bursts with Phenytoin: Case Report. Clinical EEG (Electroencephalography), 17, 173-175.

[21] Treiman, D.M. (1987) Status Epilepticus. In: Johnson, T.R., Ed., Current Therapy in Neurologic Disease, Vol. 2, Decker, New York, 38-42. 